\title{
Geoelectric imaging properties of traditional arrays and of the optimized Stummer configuration
}

\author{
S. Szalai*, A. Koppán, K. Szokoli and L. Szarka
}

Received August 2011, revision accepted October 2012

\begin{abstract}
In this paper a systematic, semi-empirical comparison is presented between two-dimensional geoelectric models and their inversion images, obtained by using five different electrical resistivity arrays and an optimized Stummer configuration. Eight different models (more or less in order of growing complexity) are studied and both noise-free and noisy data cases are considered. The results show that (1) the quality of the inversion images obtained with traditional arrays depends significantly on the model and on the noise level, (2) among the traditional arrays it is definitely the dipole-dipole array that provides inversion images mostly similar to the geoelectric models, (3) the inversion images obtained by using the optimized Stummer configuration are even more similar to the original geoelectric model than those obtained by the dipole-dipole array. It means that the optimized Stummer array is even better than the best traditional array, the dipole-dipole array, especially in the deepest part of the inversion images. We conclude that in a general field situation the Stummer configuration is good enough for not being forced to search specific configurations. As presented, optimization procedures, involving null arrays could even further improve the quality of the inversion images obtained by using the Stummer configuration.
\end{abstract}

\section{INTRODUCTION}

Geoelectric methods form a traditional group of geophysical techniques (Van Nostrand and Cook 1966; Alpin et al. 1966; Zhdanov and Keller 1993). In the early times their use was restricted to mineral exploration. Nowadays they are frequently applied in numerous field problems (Butler 2005), related to electrical resistivity distribution of the subsurface: hydrogeology (Kirsch 2006), environmental studies (Ward 1990; Knödel et al. 2005), engineering (Ward 1990) and archaeological problems (Clark 1990), etc.

The number of published geoelectric arrays used for geoelectric measurements is more than one hundred (Szalai and Szarka 2008a). It is widely known (mainly from Ward 1990) that each array has some specific advantages and disadvantages. Studying these qualities the arrays were compared from many different aspects. One of the key parameters, the depth of the investigation value was calculated by Szalai et al. (2009) following the slightly different definitions given by Edwards (1977) and Roy and Apparao (1971) for all arrays. Szalai et al. (2009) showed as well, that another key parameter, the resolution is in general in an inverse relationship with the depth of investigation values for various arrays. Parameter sensitivity maps, which are crucial in understanding the different arrays, were presented by Szalai and Szarka (2008b,c) for all ever existed arrays. Ward (1990) evaluated the geoelectric arrays from 14 various aspects. Although the aforementioned investigations aimed at providing a theoretical

*szalai@ggki.hu basis for traditional profiling and sounding techniques they are also important for electrical resistivity tomography (ERT) measurements because the individual arrays serve as a basis for the ERT measurements.

ERT measurements, which nowadays play a dominating role in geoelectric probing, should however be handled differently from the individual arrays. In their case the DOI (depth of investigation) introduced by Oldenburg and Li (1999) and the DD (depth of detectability) introduced by Szalai et al. (2011) values can give information about the depth interval from which one is able to obtain useful information. DOI is the depth, below which any change in the model resistivity has an unobservable effect on the measured signal. The DOI is in theory array- and modeldependent but for the same model, in the case of various arrays, more or less the same DOI value is obtained. At the same time, the DD parameter shows a more significant array- and modeldependence. E.g., the same model by using a given array could be observed from even a four-five times larger depth than by using another array. We supposed a relation between the DD values of the configurations and their imaging properties. The existence of such a relation was verified in this paper.

Due to ERT measurements becoming the dominating tool in geoelectric research in the past decades, it is of crucial importance to maximize the information available using them. There are actually significant efforts to find the best possible, so-called optimized arrays. One of these optimized arrays, the Stummer configuration (Stummer et al. 2004) - in contrast to the classical configuration approach - may contain a series of very different 
arrays. The basis of the Stummer configuration is a set of 147 dipole-dipole (Dp-Dp) arrays. In the optimization process a new Dp-Dp array is added in such a way that in each step the value of the so-called goodness function should have a maximum increase. (For the definition of the goodness function see Stummer et al. 2004.) We will compare the imaging properties of the Stummer array with the imaging properties of many traditional arrays.

The optimized Stummer configuration has already been tested in the field. Stummer et al. (2004) in their field test over a waste deposit site demonstrated the priority of their optimized configuration. The Stummer configuration provided inversion images from larger depths and with more details than traditional arrays and their combinations. Martorana et al. (2009) completed their theoretical investigation with a field test by using four arrays. Nyquist et al. (2007) demonstrated that the DOI value of the Stummer configuration is larger than that of the Dp-Dp configuration. At the same time, in the field experiment they did not see any meaningful difference between the inverted results of these two configurations. The objective of this paper is among others to see whether the imaging power of the Stummer configuration is better than that of other geoelectric configurations.

In our methodology, forward modelling is first carried out for a wide range of models. Inverting the forward modelling results both without and with an added white noise of $5 \%$, it will be compared as to how much the inverted images are similar to the original models. In such a way we compare ERT imaging properties of different arrays for a wide range of subsurface twodimensional models.

A similar study comparing imaging features of different ERT configurations over two-dimensional models was carried out by Dahlin and Zhou (2004). We principally followed their methodology but our approach contains many novelties. They comprise 1) extending the survey to the optimized Stummer configuration, which - as it will be shown - has key importance; 2) considering more and different models; 3 ) providing a qualification, based on quantitative parameters; 4) showing how the imaging properties of each configuration are related to the depth of detectability; 5) revealing new directions for the optimization.

\section{THE METHOD}

We studied how the various configurations are able to reconstruct the subsurface model. At first forward modelling was carried out and then noise was added to the synthetic data. The random values were generated with Gaussian distribution (i.e., with the mean value equal to zero and the standard deviation equal to $0 \%$ and $5 \%$ of the unperturbed resistivity values). The obtained data were then inverted and they were compared as to how similar the original subsurface model and the inversion image were.

The inversion was carried out by using the software Earthlmager 2D Version 2.1.7 (Advanced Geosciences 2006) in each case with the same parameter setting. The software is based largely on the results by Tarantola (1987), Dey and
Morrison (1979), Lines and Treitel (1984) and Farquharson and Oldenburg (1998). The forward modelling was carried out by applying the finite-element technique (Huebner and Thornton 1995). For the inversion, we used so-called smooth model inversion, which finds the smoothest possible model whose response fits the data to an a priori Chi-squared statistic (Advanced Geosciences 2006). The so-called estimated noise (with the lack of prior knowledge of the standard deviation of the data one may assume a certain percentage of error in the data) was $0.1 \%$ and $2 \%$, depending on the applied noise level $(0 \%, 5 \%)$. The reason for the association of the estimated noise values and the applied noise levels lies in the fact that when $0 \%$ of the applied noised level was accompanied to $0 \%$ of estimated noise, in the case of some arrays (first of all for the PP and PDP ones) only one iteration was executed. At the same time, when the estimated noise was slightly altered (to $0.1 \%$ ), already several iterations had been executed and the results proved to be better. In the case of $5 \%$ of applied noise level, when we applied $5 \%$ estimated noise, the resulting model was too smoothed, we lost a lot of information. In the case of reduced estimated noise the inversion proved to be more exact. The error reduction, including that of the L2 norm, was set to 5\%. Both the damping factor and stabilizing factor were set to 1 . For all other parameters their default values were used.

In order to qualify how similar the obtained inversion image is to the original subsurface model (shown in Figs 1-8), the following criteria were considered: 1 ) if the characteristic source of the anomaly appears in the obtained inversion image, 2) how the specific geometry of the subsurface model is visible in the inversion image, 3) what is the resolution of the inversion image. The crucial point is the first one (i.e., the detectability), since without an observable effect in the inversion image any other investigation is meaningless. The detectability (criterion 1) is the basis of not only criteria 2 and 3 but it is also a basis of the correctness of positioning and of the correctness of resistivity. As found, if the anomaly was already observable in the inversion image, then it appeared more or less in the right horizontal position. The true and inverted resistivities may be very different, especially in case of small-size anomalous bodies embedded in a high-resistivity host rock. For all the three criteria parameters an empirical qualification scheme was introduced, ranging in a numerical scale from 1-10. Albeit such a procedure is more or less subjective and incomplete (for example the resolution can be classified only in case of certain models), the number, formed from the mean value of the criteria parameters, was proven to be informative and useful, because it makes possible an easy comparison of the imaging capabilities of different configurations.

Eight various model geometries were selected, some of them with low-resistivity superficial layers and/or near-surface inhomogeneities. In order to study resolution capabilities, we varied the depth of the anomalous body as well as its number. In this way, with a reasonable number of versions a wide range of possibilities was considered. 

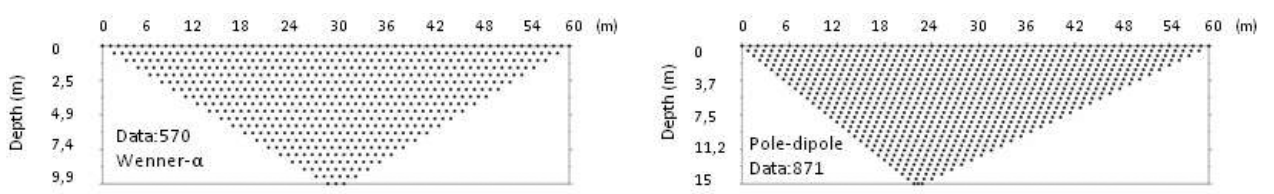

FIGURE 1

Distribution of data points and coverage for each electrode array.
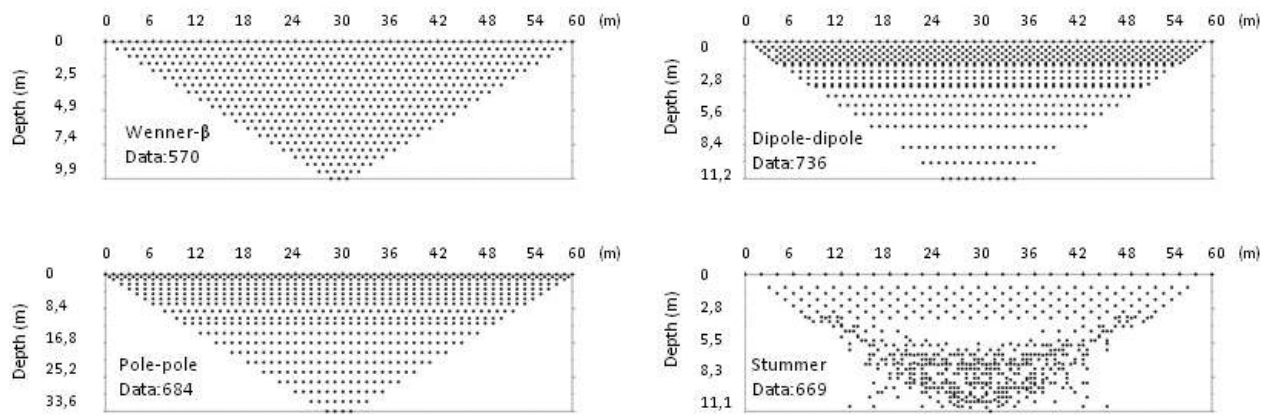

TABLE 1

RMS values from the inversion of data.

\begin{tabular}{lcccccccccccc}
\hline & \multicolumn{2}{c}{ Wenner- $\alpha$} & \multicolumn{2}{c}{ Wenner- $\beta$} & \multicolumn{2}{c}{ Pole-Pole } & \multicolumn{2}{c}{ Pole-Dipole } & \multicolumn{2}{c}{ Dipole-Dipole } & \multicolumn{2}{c}{ Stummer } \\
& $\begin{array}{c}\mathbf{0 \%} \\
\text { noise }\end{array}$ & $\mathbf{5 \%}$ & $\mathbf{0 \%}$ & $\mathbf{5 \%}$ & $\mathbf{0 \%}$ & $\mathbf{5 \%}$ & $\mathbf{0 \%}$ & $\mathbf{5 \%}$ & $\mathbf{0 \%}$ & $\mathbf{5 \%}$ & $\mathbf{0 \%}$ & $\mathbf{5 \%}$ \\
noise & noise & noise & noise & noise & noise & noise & noise & noise & noise & noise \\
\hline Model 1 & 0,1 & 4,64 & 0,1 & 4,35 & 0,1 & 4,37 & 0,1 & 4,62 & 0,34 & 4,59 & 0,11 & 4,67 \\
Model 2 & 0,1 & 4,51 & 0,33 & 4,47 & 0,34 & 4,31 & 0,12 & 4,52 & 0,63 & 4,55 & 0,3 & 4,91 \\
Model 3 & 0,14 & 4,52 & 0,29 & 4,9 & 0,11 & 4,61 & 0,27 & 4,89 & 1,99 & 4,46 & 0,39 & 4,99 \\
Model 4 & 0,09 & 4,35 & 0,1 & 4,39 & 0,1 & 4,32 & 0,09 & 4,49 & 0,56 & 4,34 & 0,16 & 4,59 \\
Model 5 & 0,16 & 4,53 & 0,3 & 4,75 & 0,13 & 4,67 & 0,29 & 4,65 & 0,91 & 4,67 & 0,31 & 4,77 \\
Model 6 & 0,13 & 4,72 & 0,38 & 4,74 & 0,54 & 4,55 & 0,22 & 4,74 & 2,1 & 4,88 & 0,3 & 4,92 \\
Model 7 & 5,12 & 5,35 & 0,97 & 4,45 & 1,4 & 4,5 & 0,66 & 4,83 & 1,08 & 5,63 & 1,13 & 5,51 \\
Model 8 & 4,29 & 4,78 & 2,04 & 4,62 & 22,59 & 8,04 & 7,54 & 4,89 & 11,13 & 5,21 & 2,81 & 5,83 \\
\hline
\end{tabular}

For this experiment, five traditional electrode configurations, which are the most frequently used in ERT measurements: Wenner- $\alpha$, Wenner- $\beta$, P-Dp (pole-dipole), P-P (pole-pole), $\mathrm{Dp}-\mathrm{Dp}$ (dipole-dipole) were selected. Among them there are two-, three- and four-electrode arrays as well, since it was found that there is a great variability in their depth of investigation and resolution values (Szalai et al. 2009) and also in their depth of detectability values (Szalai et al. 2011). In order to verify its assumed superiority, as a sixth configuration, the optimized Stummer configuration (Stummer et al. 2004) was selected.

The Wenner- $\alpha$, Wenner- $\beta$, P-Dp, Dp-Dp and P-P configurations are widely known (for a recent description and configuration details see Advanced Geosciences, Inc. 2006). By using a 60 electrode measuring system, the number of individual voltage measurements for these five configurations is 570, 570, 871, 736 and 684. In each array the maximum possible number of data points were recorded, that is the quality could not be further improved. The only exception is the Stummer array, where the number of data could still be increased. In order to get a 60 elec- trode Stummer configuration instead of its original 30 electrode version, its electrode number was doubled. We took the first 669 voltage measurements and in this way the number of Stummer configuration measurements falls in the desired 570-684 range. Figure 1 illustrates the distribution of data points and the subsurface coverage for each electrode array.

\section{RESULTS}

The original models and their inversion images, in the cases of both $(0 \%$ and $5 \%)$ noise levels, are shown in Figs 2-9. The value of the data misfit for each inversion result is shown in Table 1 for both noise levels. The root mean square (RMS) values are reasonably small, with the exception of model 8, which is the most complicated model. In the noise-free case the RMS values are in general less than 1 , while in the case of $5 \%$ noise, they fluctuate around a larger value (approximately 4).

The qualification values are summarized in Table 2. Further discussion is based on their mean values. Due to a strong correlation among parameters $1-3$, their individual interpretation is needed only in exceptional cases. 
model 1
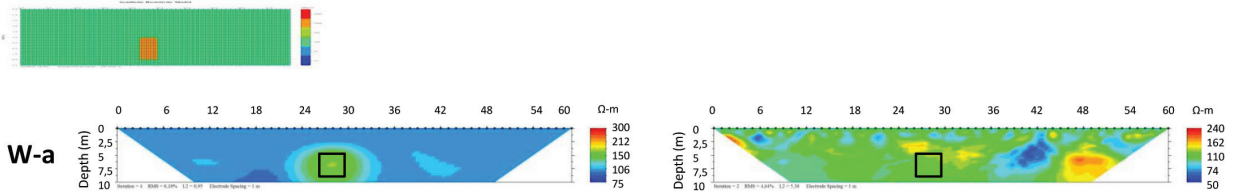
W-b
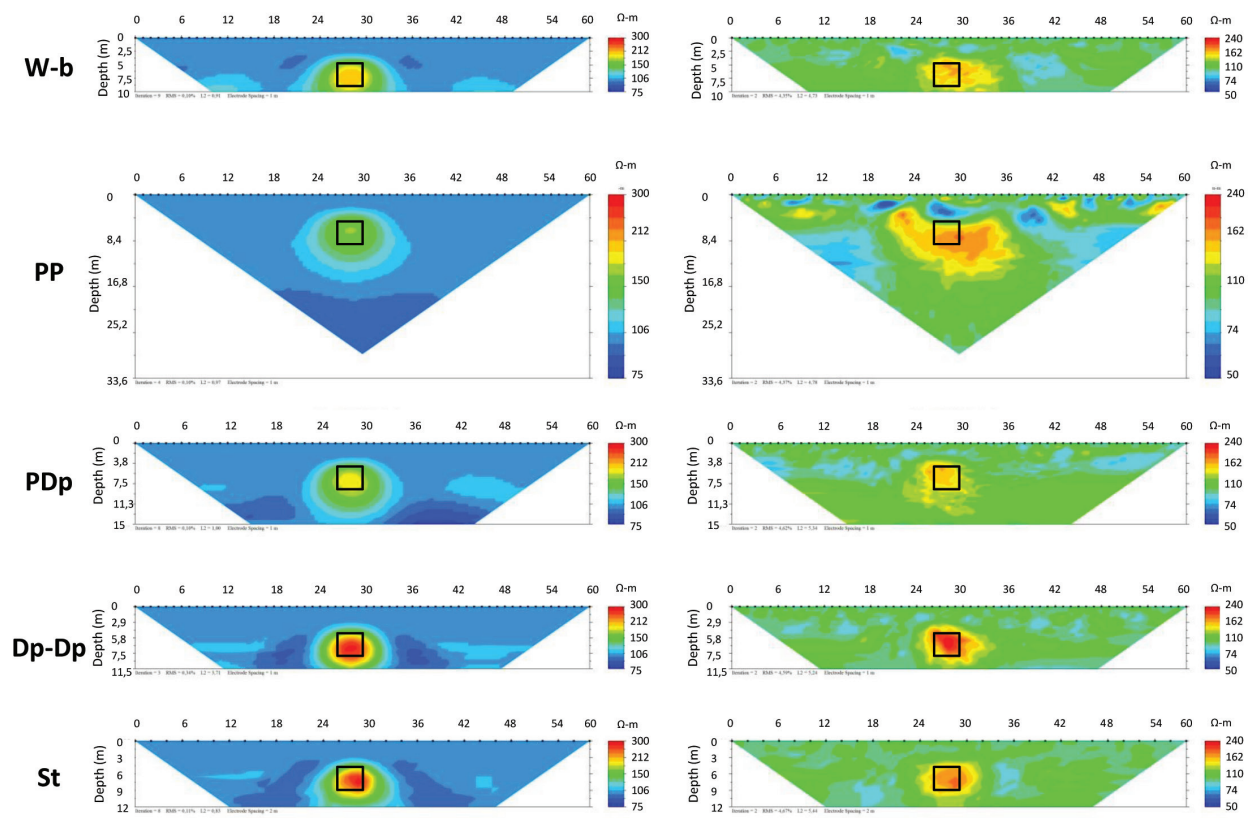

\section{model 2}
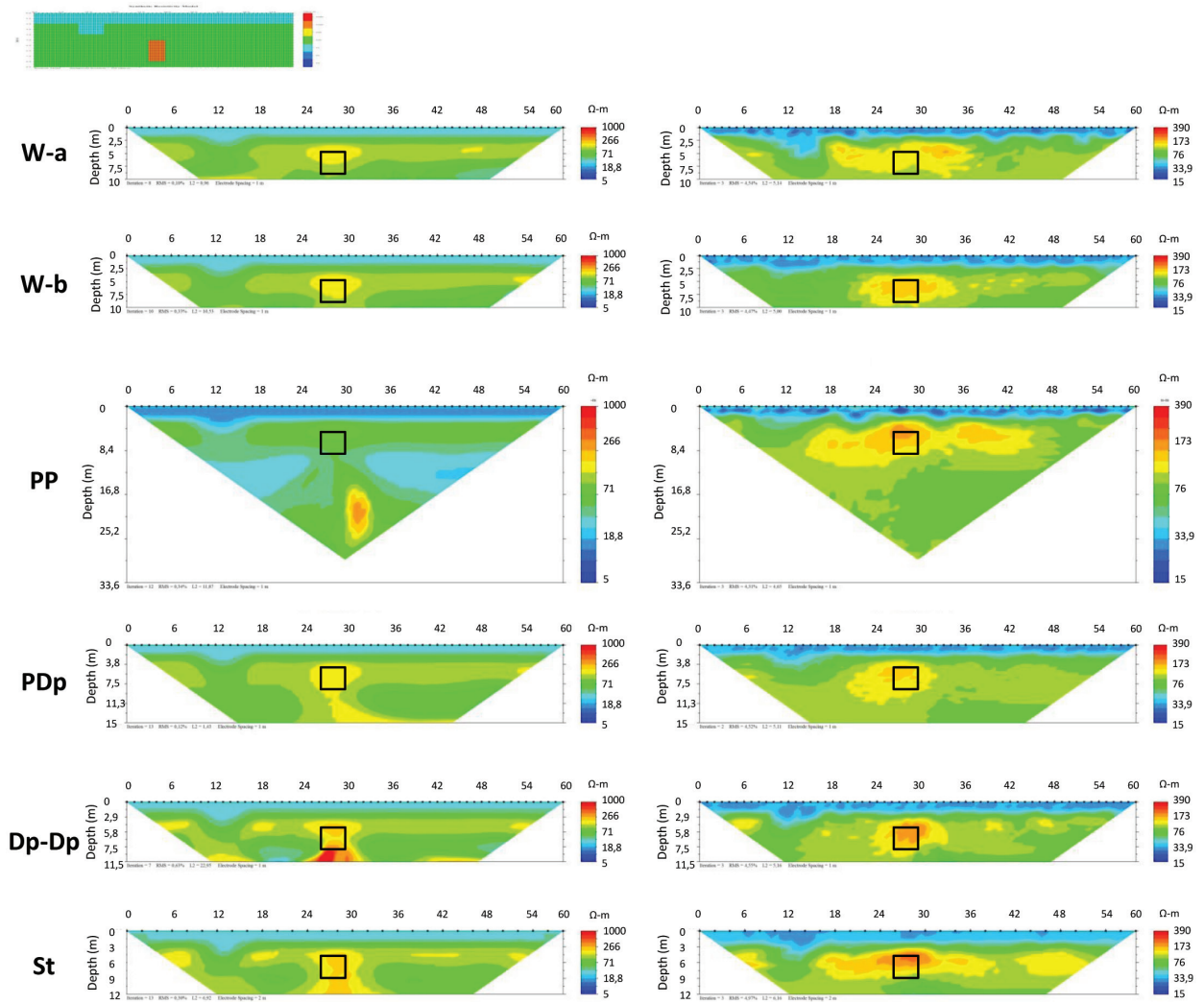

FIGURE 2

Model 1 and the inversion images from noise-free and noisy forward modelled data by using six electrode configurations.

FIGURE 3

Model 2 and the inversion images from noise-free and noisy forward modelled data by using six electrode configurations. 
model 3
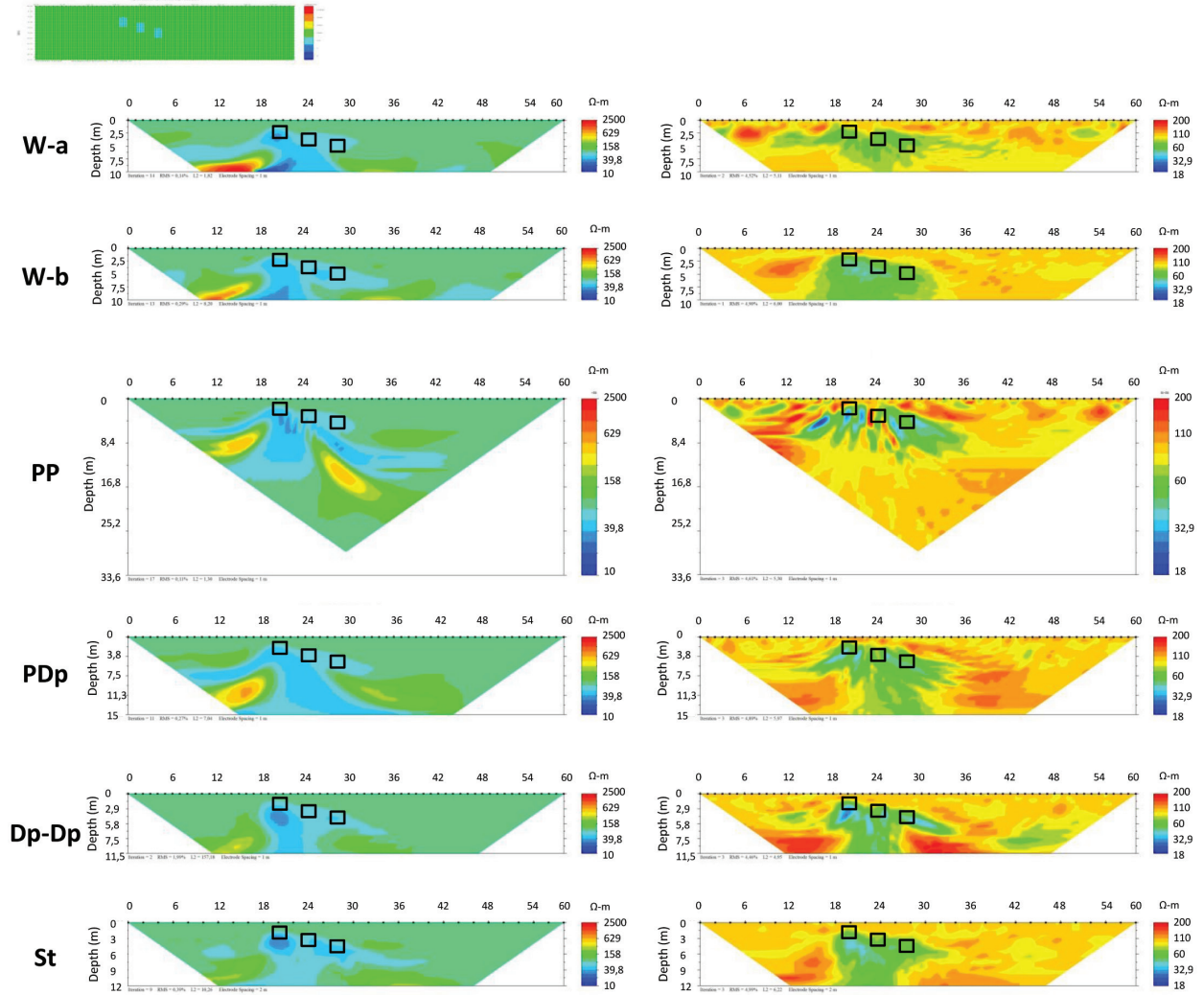

\section{model 4}
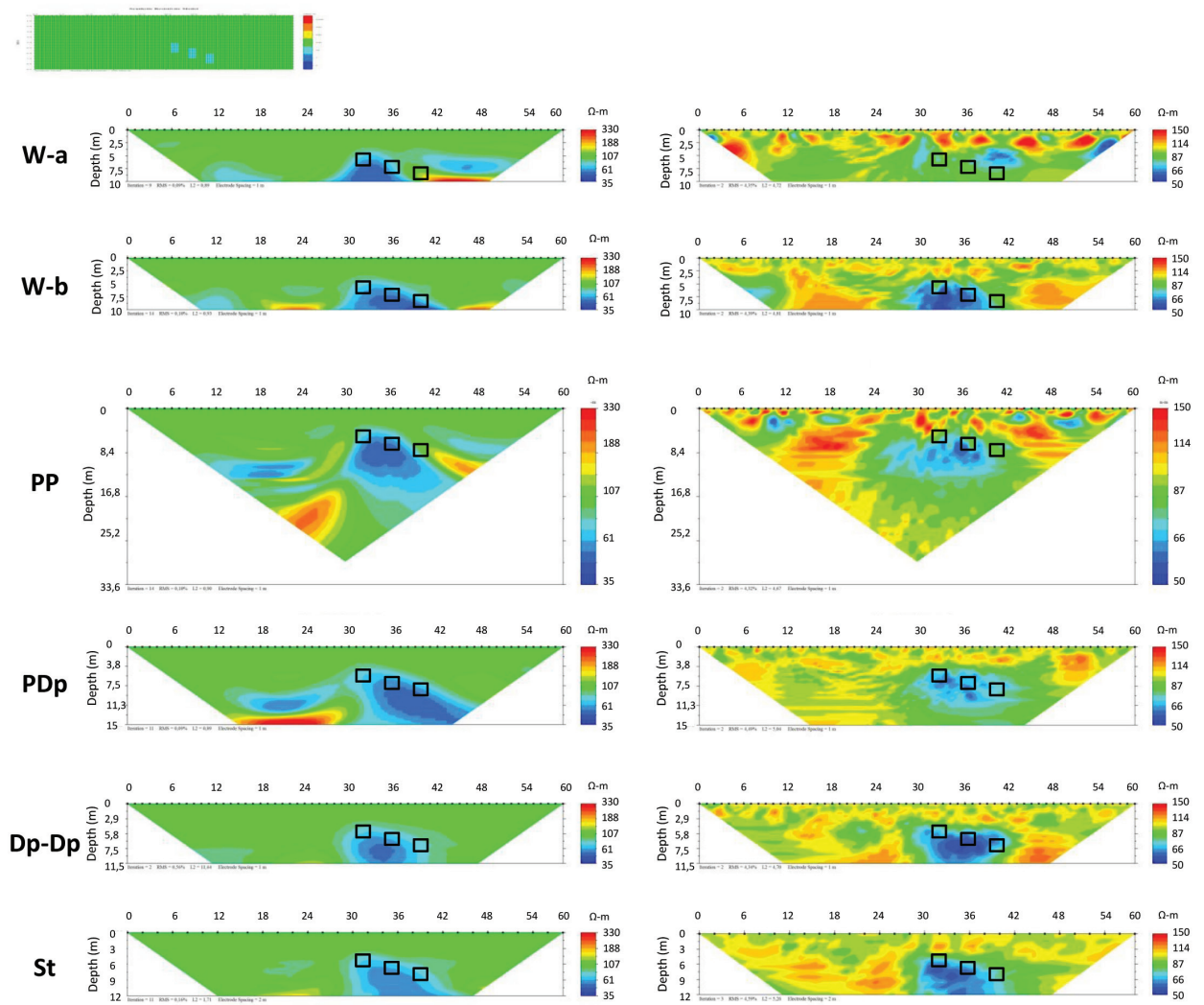

FIGURE 4

Model 3 and the inversion images from noise-free and noisy forward modelled data by using six electrode configurations.

FIGURE 5

Model 4 and the inversion images from noise-free and noisy forward modelled data by using six electrode configurations. 
model 5
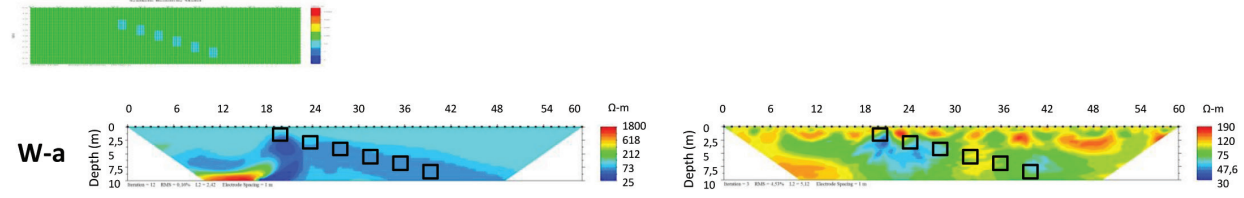

W-b
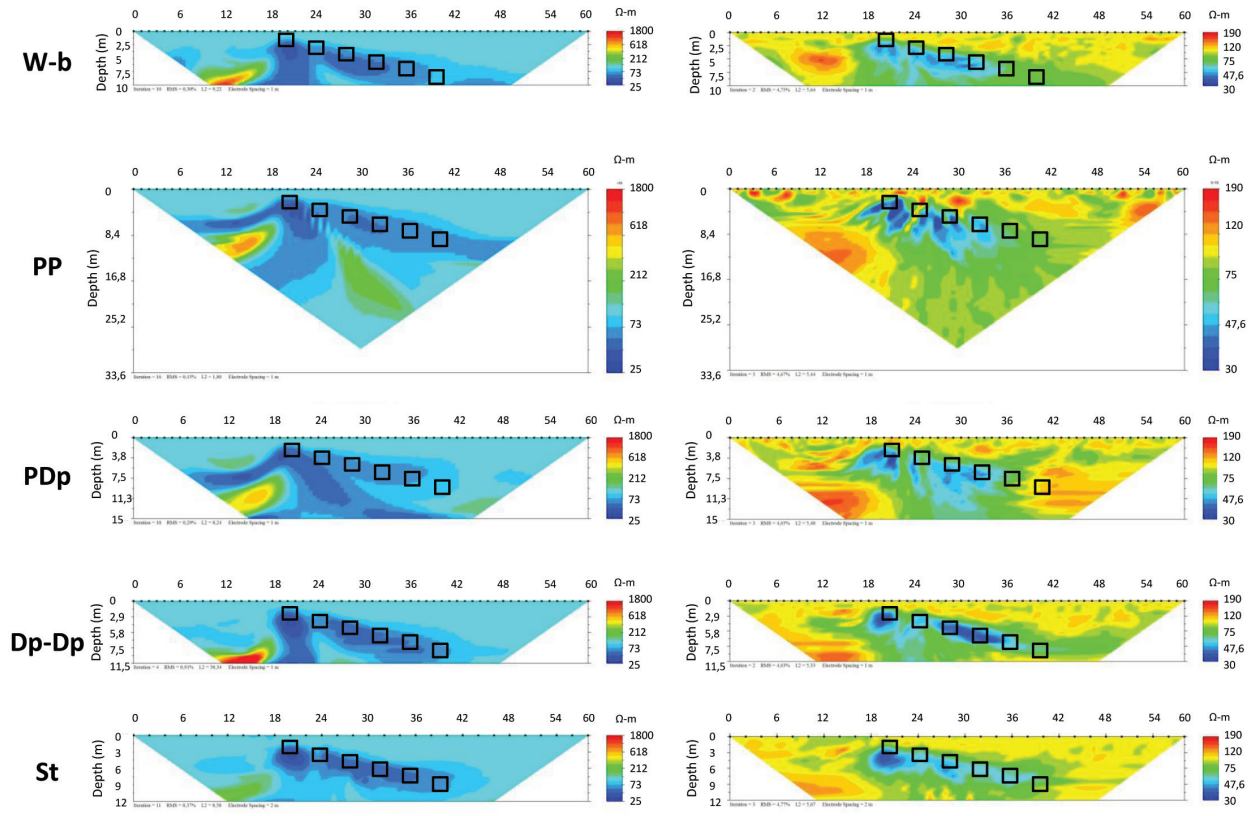

model 6
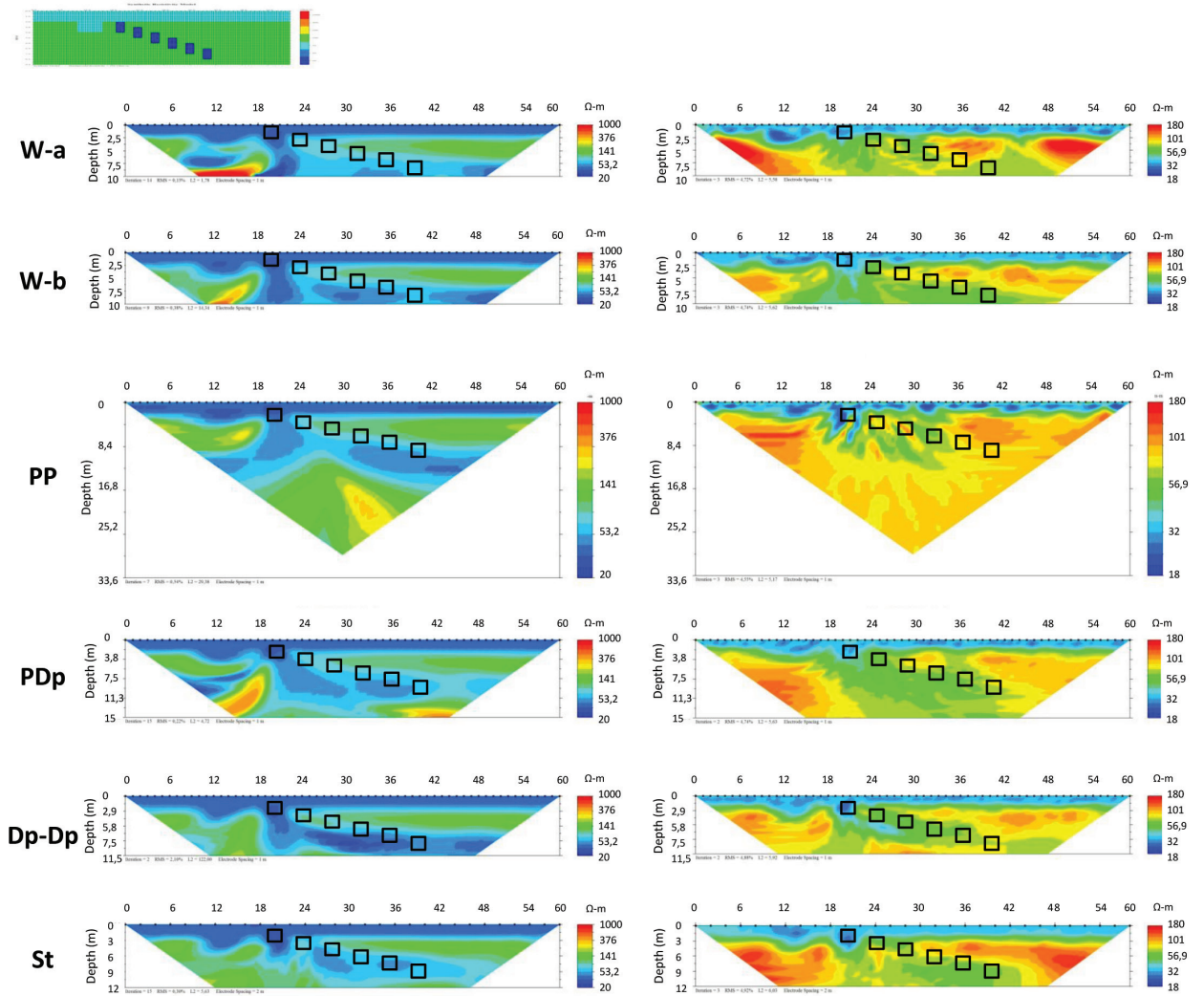

FIGURE 6

Model 5 and the inversion images from noise-free and noisy forward modelled data by using six electrode configurations.

FIGURE 7

Model 6 and the inversion images from noise-free and noisy forward modelled data by using six electrode configurations. 
model 7

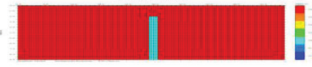

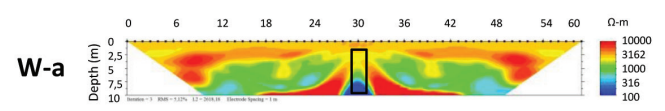

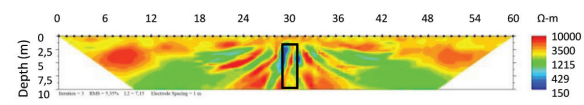
W-b
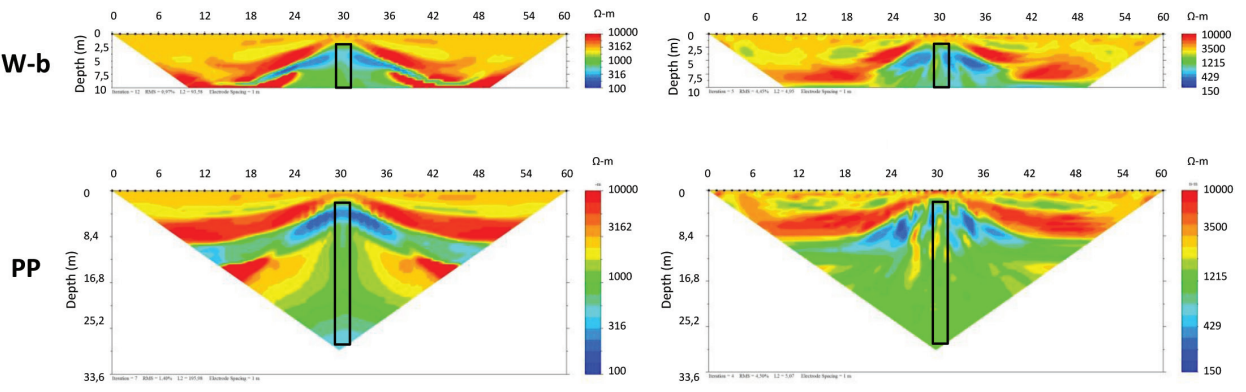

PDp
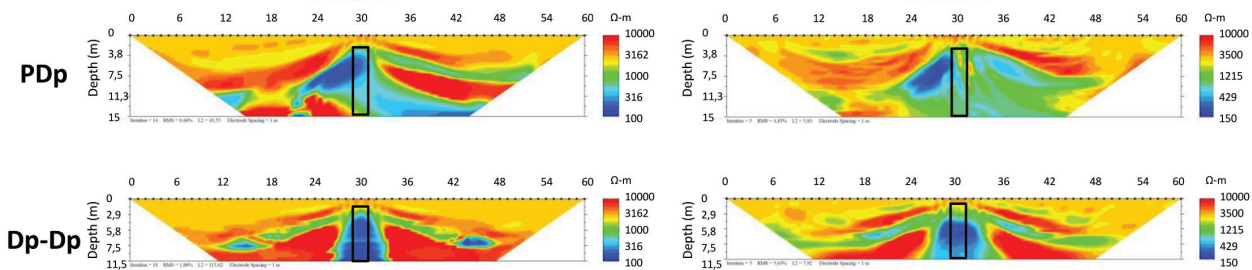

St
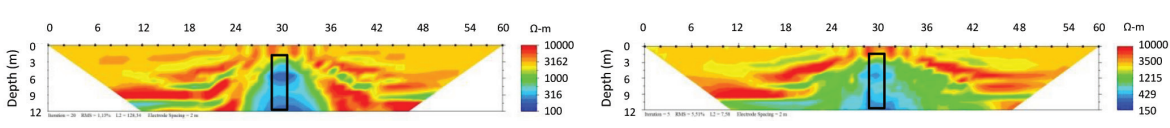

model 8
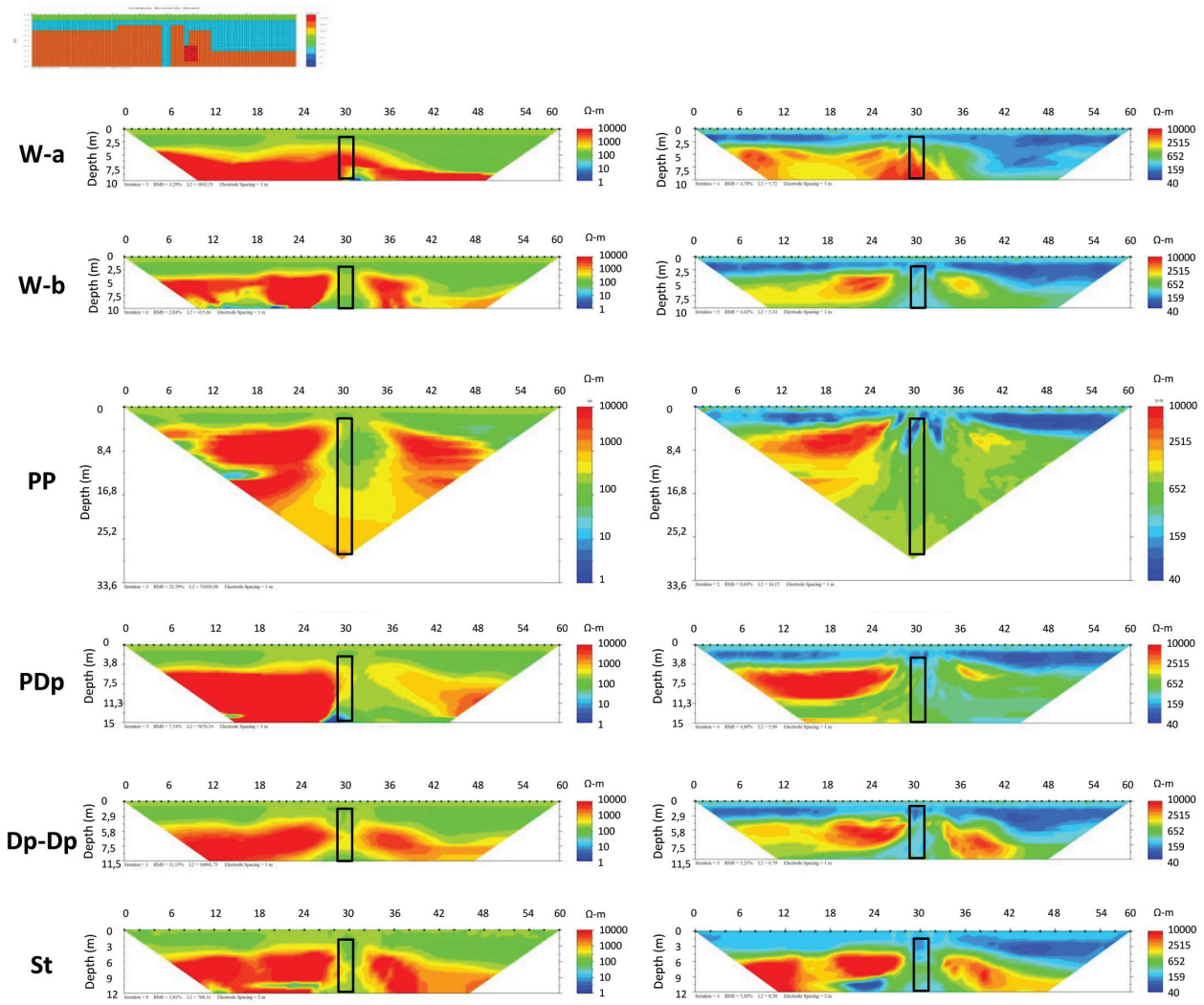

FIGURE 8

Model 7 and the inversion images from noise-free and noisy forward modelled data by using six electrode configurations.

FIGURE 9

The investigated part of model 8 and the inversion images from noise-free and noisy forward modelled data by using six electrode configurations. 
TABLE 2

Quality of inversion images obtained with six different geoelectric configurations for the eight models. The symbol * means that the parameter is irrelevant.

\begin{tabular}{|c|c|c|c|c|c|c|c|}
\hline model number & noise level & array type & $\begin{array}{l}\text { 1. detectability } \\
\text { and positioning }\end{array}$ & $\begin{array}{l}\text { 2. geometry } \\
\text { recovery }\end{array}$ & 3. resolution & $\begin{array}{c}\text { mean value of } \\
1,2 \text { and } 3\end{array}$ & $\begin{array}{c}\text { recommended } \\
\text { array }\end{array}$ \\
\hline \multirow{12}{*}{$\begin{array}{l}\dot{\bar{g}} \\
\dot{\Xi}\end{array}$} & \multirow{6}{*}{$0 \%$} & Wenner- $\alpha$ & 10 & 8 & * & 9,0 & Wenner- $\alpha$ \\
\hline & & Wenner- $\beta$ & 10 & 8 & $*$ & 9,0 & Wenner- $\beta$ \\
\hline & & PP & 10 & 7 & $*$ & 8,5 & \\
\hline & & P-Dp & 10 & 8 & $*$ & 9,0 & P-Dp \\
\hline & & Dp-Dp & 10 & 8 & $*$ & 9,0 & Dp-Dp \\
\hline & & Stummer & 10 & 8 & $*$ & 9,0 & Stummer \\
\hline & \multirow{6}{*}{$5 \%$} & Wenner- $\alpha$ & 3 & 1 & * & 2,0 & \\
\hline & & Wenner- $\beta$ & 8 & 4 & $*$ & 6,0 & \\
\hline & & $\mathrm{PP}$ & 10 & 6 & $*$ & 8,0 & \\
\hline & & P-Dp & 9 & 7 & $*$ & 8,0 & \\
\hline & & Dp-Dp & 10 & 9 & $*$ & 9,5 & Dp-Dp \\
\hline & & Stummer & 9 & 9 & $*$ & 9,0 & Stummer \\
\hline \multirow{12}{*}{$\begin{array}{l}\text { i } \\
\frac{\mathrm{d}}{\mathrm{\Xi}} \\
\mathrm{g}\end{array}$} & \multirow{6}{*}{$0 \%$} & Wenner- $\alpha$ & 7 & 8 & $*$ & 7,5 & Wenner- $\alpha$ \\
\hline & & Wenner- $\beta$ & 6 & 5 & $*$ & 5,5 & \\
\hline & & PP & 0 & 0 & $*$ & 0,0 & \\
\hline & & P-Dp & 8 & 7 & * & 7,5 & P-Dp \\
\hline & & Dp-Dp & 6 & 6 & * & 6,0 & \\
\hline & & Stummer & 8 & 6 & $*$ & 7,0 & Stummer \\
\hline & \multirow{6}{*}{$5 \%$} & Wenner- $\alpha$ & 0 & 0 & $*$ & 0,0 & \\
\hline & & Wenner- $\beta$ & 7 & 5 & * & 6,0 & \\
\hline & & $\mathrm{PP}$ & 2 & 2 & $*$ & 2,0 & \\
\hline & & P-Dp & 4 & 3 & $*$ & 3,5 & \\
\hline & & Dp-Dp & 9 & 8 & $*$ & 8,5 & Dp-Dp \\
\hline & & Stummer & 9 & 8 & $*$ & 8,5 & Stummer \\
\hline \multirow{12}{*}{ 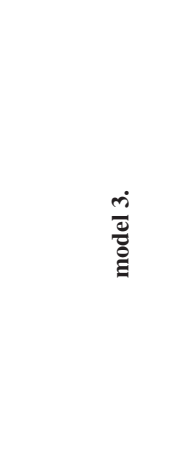 } & \multirow{6}{*}{$0 \%$} & Wenner- $\alpha$ & 5 & 6 & 2 & 4,3 & \\
\hline & & Wenner- $\beta$ & 6 & 6 & 3 & 5,0 & \\
\hline & & $\mathrm{PP}$ & 6 & 4 & 3 & 4,3 & \\
\hline & & P-Dp & 6 & 5 & 3 & 4,7 & \\
\hline & & Dp-Dp & 9 & 7 & 1 & 5,7 & Dp-Dp \\
\hline & & Stummer & 8 & 6 & 4 & 6,0 & Stummer \\
\hline & \multirow{6}{*}{$5 \%$} & Wenner- $\alpha$ & 2 & 2 & 0 & 1,3 & \\
\hline & & Wenner- $\beta$ & 4 & 4 & 3 & 3,7 & \\
\hline & & PP & 8 & 8 & 2 & 6,0 & \\
\hline & & P-Dp & 9 & 9 & 6 & 8,0 & P-Dp \\
\hline & & Dp-Dp & 10 & 8 & 5 & 7,7 & Dp-Dp \\
\hline & & Stummer & 9 & 9 & 7 & 8,3 & Stummer \\
\hline \multirow{12}{*}{ 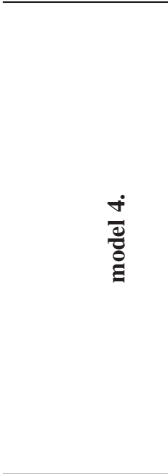 } & \multirow{6}{*}{$0 \%$} & Wenner- $\alpha$ & 8 & 4 & 0 & 4,0 & \\
\hline & & Wenner- $\beta$ & 10 & 8 & 0 & 6,0 & Wenner- $\beta$ \\
\hline & & $\mathrm{PP}$ & 7 & 8 & 0 & 5,0 & \\
\hline & & P-Dp & 7 & 9 & 0 & 5,3 & \\
\hline & & Dp-Dp & 9 & 7 & 0 & 5,3 & \\
\hline & & Stummer & 10 & 9 & 0 & 6,3 & Stummer \\
\hline & \multirow{6}{*}{$5 \%$} & Wenner- $\alpha$ & 3 & 2 & 0 & 1,7 & \\
\hline & & Wenner- $\beta$ & 9 & 6 & 0 & 5,0 & Wenner- $\beta$ \\
\hline & & PP & 8 & 8 & $\mathbf{0}$ & 5,3 & PP \\
\hline & & P-Dp & 6 & 4 & 0 & 3,3 & \\
\hline & & Dp-Dp & 8 & 5 & 0 & 4,3 & \\
\hline & & Stummer & 9 & 8 & o & 5,7 & Stummer \\
\hline
\end{tabular}




\begin{tabular}{|c|c|c|c|c|c|c|c|}
\hline model number & noise level & array type & $\begin{array}{l}\text { 1. detectability } \\
\text { and positioning }\end{array}$ & $\begin{array}{l}\text { 2. geometry } \\
\text { recovery }\end{array}$ & 3. resolution & $\begin{array}{c}\text { mean value of } \\
1,2 \text { and } 3\end{array}$ & $\begin{array}{c}\text { recommended } \\
\text { array }\end{array}$ \\
\hline \multirow{12}{*}{$\begin{array}{l}\frac{10}{\square} \\
\frac{\tilde{g}}{0} \\
\Xi\end{array}$} & \multirow{6}{*}{$0 \%$} & Wenner- $\alpha$ & 8 & 7 & 0 & 5,0 & \multirow{4}{*}{ Wenner- $\beta$} \\
\hline & & Wenner- $\beta$ & 9 & 8 & 3 & 6,7 & \\
\hline & & $\mathrm{PP}$ & 9 & 8 & 1 & 6,0 & \\
\hline & & P-Dp & 8 & 7 & 1 & 5,3 & \\
\hline & & Dp-Dp & 10 & 8 & 3 & 7,0 & Dp-Dp \\
\hline & & Stummer & 10 & 10 & 5 & 8,3 & Stummer \\
\hline & \multirow{6}{*}{$5 \%$} & Wenner- $\alpha$ & 7 & 6 & 2 & 5,0 & \\
\hline & & Wenner- $\beta$ & 9 & 8 & 0 & 5,7 & \\
\hline & & $\mathrm{PP}$ & 6 & 7 & 0 & 4,3 & \\
\hline & & P-Dp & 6 & 6 & 1 & 4,3 & \\
\hline & & Dp-Dp & 7 & 5 & 2 & 4,7 & \\
\hline & & Stummer & 10 & 9 & 4 & 7,7 & Stummer \\
\hline \multirow{12}{*}{ 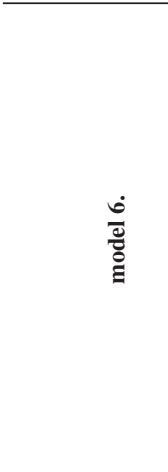 } & \multirow{6}{*}{$0 \%$} & Wenner- $\alpha$ & 1 & 0 & 0 & 0,3 & \multirow{5}{*}{ P-Dp } \\
\hline & & Wenner- $\beta$ & 0 & 0 & 0 & 0,0 & \\
\hline & & $\mathrm{PP}$ & 5 & 4 & 0 & 3,0 & \\
\hline & & P-Dp & 7 & 4 & 2 & 4,3 & \\
\hline & & Dp-Dp & 1 & 1 & 0 & 0,7 & \\
\hline & & Stummer & 5 & 5 & 2 & 4,0 & Stummer \\
\hline & \multirow{6}{*}{$5 \%$} & Wenner- $\alpha$ & 2 & 2 & 0 & 1,3 & \multirow{6}{*}{ Dp-Dp } \\
\hline & & Wenner- $\beta$ & 5 & 5 & 0 & 3,3 & \\
\hline & & $\mathrm{PP}$ & 4 & 4 & 0 & 2,7 & \\
\hline & & P-Dp & 4 & 4 & 0 & 2,7 & \\
\hline & & Dp-Dp & 6 & 6 & 0 & 4,0 & \\
\hline & & Stummer & 4 & 4 & 0 & 2,7 & \\
\hline \multirow{12}{*}{ 힐 } & \multirow{6}{*}{$0 \%$} & Wenner- $\alpha$ & 4 & 4 & * & 4,0 & \multirow{6}{*}{$\begin{array}{c}\text { Dp-Dp } \\
\text { Stummer }\end{array}$} \\
\hline & & Wenner- $\beta$ & 2 & 2 & * & 2,0 & \\
\hline & & PP & 4 & 2 & $*$ & 3,0 & \\
\hline & & P-Dp & 2 & 1 & $*$ & 1,5 & \\
\hline & & Dp-Dp & 10 & 8 & $*$ & 9,0 & \\
\hline & & Stummer & 10 & 7 & $*$ & 8,5 & \\
\hline & \multirow{6}{*}{$5 \%$} & Wenner- $\alpha$ & 2 & 2 & * & 2,0 & \multirow{6}{*}{$\begin{array}{c}\text { Dp-Dp } \\
\text { Stummer }\end{array}$} \\
\hline & & Wenner- $\beta$ & 7 & 2 & * & 4,5 & \\
\hline & & $\mathrm{PP}$ & 3 & 2 & * & 2,5 & \\
\hline & & P-Dp & 6 & 2 & $*$ & 4,0 & \\
\hline & & Dp-Dp & 10 & 8 & * & 9,0 & \\
\hline & & Stummer & 9 & 6 & $*$ & 7,5 & \\
\hline \multirow{12}{*}{ 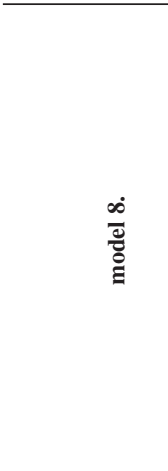 } & \multirow{6}{*}{$0 \%$} & Wenner- $\alpha$ & 4 & 5 & 4 & 4,3 & \multirow[b]{6}{*}{ Stummer } \\
\hline & & Wenner- $\beta$ & 4 & 4 & 6 & 4,7 & \\
\hline & & $\mathrm{PP}$ & 3 & 3 & 3 & 3,0 & \\
\hline & & P-Dp & 5 & 5 & 5 & 5,0 & \\
\hline & & Dp-Dp & 5 & 4 & 3 & 4,0 & \\
\hline & & Stummer & 7 & 7 & 7 & 7,0 & \\
\hline & \multirow{6}{*}{$5 \%$} & Wenner- $\alpha$ & 3 & 3 & 1 & 2,3 & \multirow{4}{*}{ Wenner- $\beta$} \\
\hline & & Wenner- $\beta$ & 6 & 6 & 6 & 6,0 & \\
\hline & & $\mathrm{PP}$ & 3 & 3 & 1 & 2,3 & \\
\hline & & P-Dp & 5 & 4 & 2 & 3,7 & \\
\hline & & Dp-Dp & 10 & 7 & 5 & 7,3 & Dp-Dp \\
\hline & & Stummer & 6 & 6 & 6 & 6,0 & Stummer \\
\hline
\end{tabular}


For model 1, in the noise-free case, as expected, each configuration provided good results, both in terms of detectability and model geometry. In the case of noisy data, the inversion images became worse, especially for the Wenner- $\alpha$ array. In the case of other arrays the inversion images are still acceptable but the noise amplifies and extends the false pseudo-anomalies. The true geometry of the subsurface model is reflected the best in the Dp-Dp and Stummer inversion images.

In the case of model 2 (i.e., in the presence of a low-resistivity superficial layer and a near-surface lateral inhomogeneity), in the noisy case, the effect of the model prism cannot already be so easily separated from the noise. The P-P array in this case is practically useless. For the other configurations the vertical delineation seems to be the greatest problem. In terms of horizontal delineation the Stummer and P-Dp configurations seem to be the best ones. With the exception of the Stummer configuration, a false, more or less continuous, horizontal layer appears in the inversion image, which is simply misleading. In the noisefree case it is difficult to tell if the Wenner- $\alpha$, P-Dp or Stummer configuration is the best one. At the same time, in the noisy case, the Dp-Dp and Stummer configurations provide the two best images. It is again the Stummer configuration that should be first of all recommended.

For model 3, in the noise-free case, the anomalous bodies were detected with each configuration but the ambiguity in vertical delineation (especially beneath the body at the smallest depth) might make the interpretation difficult. This distorting effect is the least significant in the case of Dp-Dp and Stummer configurations; consequently they are the best in terms of detectability. In terms of resolution, the Stummer, Wenner- $\beta$ and P-Dp should be perhaps mentioned. It is surprising that the Stummer, Dp-Dp, P-Dp and PP inversion images seem to be better in case of noisy data than in the case of noise-free data: the pseudo-anomalies are somehow reduced due to the noise. For model 3, the Dp-Dp, Stummer and P-Dp configurations are the most useful ones.

In the case of model 4 (the 'deep' version of model 3) the detectability improves but the resolution deteriorates. In the case of noise-free data, the Stummer and Wenner- $\beta$ arrays are the two best ones. Adding noise to the measured data, the inversion images become somewhat worse, with the exception of the PP array. For model 4, the Stummer and PP arrays are recommended.

For model 5, it is again the infinitely downward extended resistivity zone in the inversion images that is the most problematic phenomenon. While the Dp-Dp and Stummer configurations are free of this effect and their horizontal resolution is sufficiently good, these two arrays are outstanding among all the investigated arrays. Adding some noise, there is a slight deterioration in the Dp-Dp image. Therefore it is again the Stummer configuration that remains outstanding.

In the case of model 6 (in presence of a low-resistivity superficial layer), the quality of Wenner- $\alpha$, Wenner- $\beta$ and P-P inversion images significantly deteriorates already in the case of noise-free data and there is also some deterioration in the Stummer and P-Dp inversion images. At the same time, the Dp-Dp inversion images still show the same quality as for model 5. By using the latter three configurations, it is easier to separate the anomalous bodies. Again, in the case of noisy data, in a few instances even some improvement in the inversion images was observed.

For model 7, with the exception of the Dp-Dp and Stummer configurations, quite misleading inversion images were obtained. In the case of noisy data the Stummer inversion image becomes wider, therefore less useful than in the case of noise-free data. For model 7, at first the Dp-Dp array, then the Stummer configuration is recommended.

In the case of model 8 (which is the most complicated model) the topography of the resistive basement was quite well recovered with each configuration (with the exception of P-P) but the low-resistivity anomalous body (fracture/sinkhole) is seen only in the Stummer inversion images. In the case of noisy data, the high-resistivity hole (under the second 'fracture' from left to the right) is seen the best with the Stummer configuration.

\section{INTERPRETATION}

The $\beta$-type configurations (as with Dp-Dp, Wenner- $\beta$ and P-Dp) are more effective than the Wenner- $\alpha$ and PP ones (see Table 3). In light of this fact, it is not at all surprising that the Stummer configuration is superior to any of the traditional arrays, since in the first 669 measurements it applies only $\beta$-type arrays.

TABLE 3

Summary of the usefulness of the configurations for geoelectric models 1-8, shown in Figs 1-8.

\begin{tabular}{|c|c|c|c|c|c|c|c|c|c|c|c|c|c|c|c|c|c|}
\hline Array type & m1 & m1 & $\mathrm{m} 2$ & $\mathrm{~m} 2$ & m3 & m3 & m4 & $\mathrm{m} 4$ & m5 & $\mathrm{m} 5$ & m6 & m6 & m7 & m7 & m8 & $\mathrm{m} 8$ & Total number \\
\hline & $0 \%$ & $5 \%$ & $0 \%$ & $5 \%$ & $0 \%$ & $5 \%$ & $0 \%$ & $5 \%$ & 0\% & $5 \%$ & 0\% & $5 \%$ & 0\% & & & & \\
\hline Wenner- $\alpha$ & $\mathrm{x}$ & & $\mathrm{x}$ & & & & & & & & & & & & & & 2 \\
\hline Wenner- $\beta$ & $\mathrm{x}$ & & & & & & $\mathrm{x}$ & $\mathrm{x}$ & $\mathrm{x}$ & & & & & & & $\mathrm{x}$ & 5 \\
\hline PP & & & & & & & & $\mathrm{x}$ & & & & & & & & & 1 \\
\hline P-Dp & $\mathrm{x}$ & & $\mathrm{x}$ & & & $\mathrm{x}$ & & & & & $\mathrm{x}$ & & & & & & 4 \\
\hline Dp-Dp & $\mathrm{x}$ & $\mathrm{x}$ & & $\mathrm{x}$ & $\mathrm{x}$ & $\mathrm{x}$ & & & $\mathrm{x}$ & & & $\mathrm{x}$ & $\mathrm{x}$ & $\mathrm{x}$ & & $\mathrm{x}$ & 10 \\
\hline Stummer & $\mathrm{x}$ & $\mathrm{x}$ & $\mathrm{x}$ & $\mathrm{x}$ & $\mathrm{x}$ & $\mathrm{x}$ & $\mathrm{x}$ & $\mathrm{x}$ & $\mathrm{x}$ & $\mathrm{x}$ & $\mathrm{x}$ & & $\mathrm{x}$ & $\mathrm{x}$ & $\mathrm{x}$ & $\mathrm{x}$ & 15 \\
\hline
\end{tabular}


Another very important reason of the Stummer configuration's superiority is that it provides realistic images even at larger depths. This feature is a simple consequence of the fact that in the Stummer configuration the number of arrays having a large depth of investigation was increased. On the basis of the DOI concept (Oldenburg and Li 1999) one might assume that there is no need for arrays of large depth of investigation. Figures 1-8 made it clear that these arrays have a crucial role in the stabilization and even in the formation of the deepest part of the inversion images. In the case of vertical models (models 7 and 8) the bad quality of the inversion images is directly due to distortions at their deepest part.

Even larger depths could however be reached e.g., by using null- and/or quasi-null arrays (Szalai et al. 2002, 2004). These arrays are able to give well detectable signals in spite of their geometry factors (i.e., their $k$ values) being significantly larger than 5000. Such arrays were not included into the optimization procedure by Stummer et al. (2004).

A further possible direction for the optimization would be to include three-electrode arrays. It was observed by Candansayar (2008) that a joint inversion of the so-called PD-L, PD-R and DD ensemble gives better results than the single use of any electrode array. In the depth of detectability investigations by Szalai et al. (2011) the P-Dp configuration proved to be for many models even better than the dipole one. A recent attempt to optimize pole-bipole configurations has been published by Blome et al. (2011). It should not be forgotten that the arsenal of possible geoelectric arrays is immense (Szalai and Szarka 2011). Some of these arrays could be built into the optimization procedure. The original idea, realized in the construction of new arrays (Szalai and Szarka 2011), is also worth considering. Using various arrays in the same configuration makes it possible, among others, a combined weighted inversion (Athanasiou et al. 2007) or socalled object oriented focusing (Hennig et al. 2008). Both these attempts may mean further possibilities in developing the ERT technique.

It is worthwhile mentioning that, similar to these investigations, in the depth of detectability study by Szalai et al. (2011) the Dp-Dp, Wenner- $\beta$ and P-Dp configurations proved to be the best ones among six arrays (P-Dp, Wenner- $\beta$, Dp-equatorial, Wenner- $\alpha$, Dp-axial=Dp-Dp and P-P). It confirms our assumption that the crucial parameter of the imaging among all of them is detectability.

\section{CONCLUSIONS}

The inversion images obtained for eight two-dimensional models from forward-modelled noise-free and noisy data, obtained with six different electrode configurations were compared to true geoelectric models. On the basis of a qualification system, we concluded that:

a) The most important parameter that first of all determines the quality of the inversion image is detectability. All other imaging parameters are constrained by the detectability. b) Regarding traditional configurations, the $\beta$-type ones (Dp-Dp, $\mathrm{P}-\mathrm{Dp}$ and Wenner- $\beta$ ) proved to be more effective than the Wenner- $\alpha$ and P-P arrays and the Dp-Dp arrays proved to be far more effective than any other traditional array among the investigated ones.

c) The optimized Stummer configuration, which is based on systematically selected $\beta$-type arrays, proved to be far more effective for almost every model, in the case of both noise-free and noisy data, than the traditional arrays. It was only the Dp-Dp configuration that was able to compete with it. The imaging capacity of the optimized Stummer configuration is more effective than that of traditional configurations not only for small size inhomogeneities (which was already demonstrated numerically by Stummer et al. 2004) but more or less all models, including complicated ones. The optimized Stummer configuration is generally recommended among every field condition. The optimized Stummer configuration is so good that there is no need for searching field-adaptive arrays.

d) It has been shown that the arrays having a large depth of investigation play a crucial role in the improved quality of the inversion images of the optimized configuration in spite of them giving information from depths far below the corresponding DOI value. On this basis we think that the so-called 'optimized Stummer configuration' is not yet optimized. We recommend first of all involving in the selection process arrays with even a larger depth of investigation.

e) Due to the fact that a larger depth of investigation is obtained due to null- and quasi-null arrays or involving three-electrode arrays, all these arrays might further increase the imaging effectivity of the Stummer configuration. Possible other directions of further optimization of the ERT technique might be as follows: combined weighted inversion, object oriented focusing and a systematic investigation of the minimum number of arrays that are able to provide useful data.

As a summary, in terms of the quality of the inversion image the Stummer configuration proved to be more efficient than any other traditional configuration, regardless of the model and noise level. It is expected that with the planned new directions the efficiency of ERT measurements can be even further improved.

\section{ACKNOWLEDGEMENT}

The authors are grateful to Professor Farquharson and the anonymous reviewer whose recommendations improved the manuscript greatly. Sándor Szalai is a grantee of the Bolyai János Scholarship Program.

\section{REFERENCES}

Advanced Geosciences, Inc. 2006. Instruction manual for EarthImager 2D, Version 2.1.7, Resistivity and IP inversion software.

Alpin L.M., Berdichevskii M.N., Vedrintsev G.A. and Zagarmistr A.M. 1966. Dipole methods for measuring earth conductivity, Consultants Bureau, New York, p. 302. 
Athanasiou E.N., Tsourlos P.I., Papazachos C.B. and Tsokas G.N. 2007. Combined weighted inversion of electrical resistivity data arising from different data types. Journal of Applied Geophysics 62, 124-140.

Blome M., Maurer H. and Greenhalgh S. 2011. Geoelectric experimental design - Efficient acquisition and exploitation of complete pole-bipole data sets. Geophysics 76(1), F15-F26.

Butler D.K. 2005. Near-Surface Geophysics. SEG, Tulsa, Oklahoma, USA, p. 355.

Candansayar M.E. 2008. Two-dimensional individual and joint inversion of three- and four-electrode array DC resistivity data. Journal of Geophysics and Engineering 5, 290-300, doi:10.1088/17422132/5/3/005

Clark A.J. 1990. Seeing Beneath the Soil. B.T. Batshford Ltd. London, p. 176.

Dahlin T. and Zhou B. 2004. A numerical comparison of 2D resistivity imaging with 10 electrode arrays. Geophysical Prospecting 52, 379398.

Dey A. and Morrison H.F. 1979. Resistivity modeling for arbitrarily shaped two-dimensional structures. Geophysical Prospecting 27, 106-136.

Edwards L.S. 1977. A modified pseudosection for resistivity and induced-polarization. Geophysics 42, 1020-1036.

Farquharson C.G. and Oldenburg D.W. 1998. Non-linear inversion using general mesures of data misfit and model structure. Geophysical Journal International 134, 213-227.

Hennig T., Webber A. and Möller M. 2008. Object oriented focussing of geoelectrical multielectrode measurements. Journal of Applied Geophysics 65, 57-64.

Huebner K.H. and Thornton E.A. 1995. The Finite Element Method for Engineers. John Wiley and Sons, p. 744.

Kirsch R. 2006. Groundwater Geophysics. Springer, p. 493.

Knödel K., Krummel H. and Lange G. 2005. Band 3. Handbuch zur Erkundung des Untergrundes von Deponien und Altlasten, 2nd ed. Springer, p. 128.

Lines L.R. and Treitel S. 1984. A review of least squares inversion and its applications to geophysical problems. Geophysical Prospecting 32, 159-186.

Martorana R., Fiandaca G., Casas A.P. and Cosentino P.L. 2009. Comparative tests on different multi-electrode arrays using models in near-surface geophysics. Journal of Geophysics and Engineering 6, $1-20$.

Nyquist J.E., Peake J. S. and Roth M.J.S. 2007. Case History; Comparison of an opzimised resistivity array with dipole-dipole soundings in karst terrain. Geophysics 72(4), 139-144.
Oldenburg D.W. and Li Y. 1999. Estimating depth of investigation in DC resistivity and IP surveys. Geophysics 64, 403-416.

Roy A. and Apparao A. 1971. Depth of investigation in direct current methods. Geophysics 36, 943-959.

Stummer P., Maurer H. and Green A.G. 2004. Experimental design: Electrical resistivity data sets that provide optimum subsurface information. Geophysics 69(1), 120-139.

Szalai S., Novák A. and Szarka L. 2009. Depth of Investigation and Vertical Resolution of Surface Geoelectric Arrays. Journal of Environmental and Engineering Geophysics 14(1), 15-23.

Szalai S., Novák A. and Szarka L. 2011. Which geoelectric array sees the deepest in noisy environment? Depth of detectability values of multielectrode systems over various two-dimensional models. Physics and Chemistry of the Earth 36, 1398-1404, doi:10.1016/j.pce.2011.01.008

Szalai S. and Szarka L. 2008a. On the classification of surface geoelectric arrays. Geophysical Prospecting 56, 159-175.

Szalai S. and Szarka L. 2008b. Parameter sensitivity maps of surface geoelectric arrays. Part 1: Linear arrays. Acta Geodaetica et Geophysica Hungarica 43, 419-437.

Szalai S. and Szarka L. 2008c. Parameter sensitivity maps of surface geoelectric arrays. Part 2: Nonlinear and focussed arrays. Acta Geodaetica et Geophysica Hungarica 43, 439-447.

Szalai S. and Szarka L. 2011. New perspectives for two-dimensional multielectrode measurements, on basis of once-developed geoelectric arrays. Journal of Applied Geophysics 75, 1-8, doi: 10.1016/j.jappgeo.2011.06.020

Szalai S., Szarka L., Marquis G., Sailhac P., Kaikkonen P. and Lahti I. 2004. Co-linear null arrays in geoelectrics. IAGA WG 1.2 on Electromagnetic Induction in the Earth. Proceedings of the 17th Workshop, http://www.emindia2004.org

Szalai S., Szarka L., Prácser L., Bosch F., Müller I. and Turberg P. 2002. Geoelectric mapping of near-surface karstic fractures by using null arrays. Geophysics 67, 1769-1778.

Tarantola A. 1987. Inverse Problem Theory: Methods for Data Fitting and Model Parameter Estimation. Elsevier.

Van Nostrand R.G. and Cook K.L. 1966. Interpretation of resistivity data. Geological Survey Professional Paper 499, United States Government Printing Office, Washington, p. 310.

Ward S.H. 1990. Geotechnical and environmental geophysics, Vol. I. Review and tutorial. SEG, Tulsa, Oklahoma, p. 389.

Zhdanov M.S. and Keller G.V. 1993. The Geoelectrical Methods in Geophysical Exploration. Elsevier, p. 873.

\section{EAGE EURopan
ASSOCLATION OF

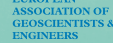 22-26 April 2013 - Gelendzhik, Russia Engineering Geophysics 2013}

Working language of the Conference is Russian!
Register now! www.eage.ru www.eage.org 